\title{
Photometric design of color-conversion LEDs
}

Hilmi Volkan Demir, Talha Erdem, Xiao Wei Sun, and Sedat Nizamoglu

Selected combinations of narrow-emitter nanophosphors, made of colloidal quantum-dots, are integrated into LEDs to achieve photometrically efficient white light sources.

Traditional broad-emitter phosphors are made by incorporating a combination of rare earth ions into ceramic powders. Although these types of phosphors are good for photon conversion across a broad spectrum, fundamental problems related to their photometric performance still persist. In particular, there are issues associated with a set of broad and continuous emissions that happen at long wavelengths. These emissions are usually accompanied by a tail, and they are scattered by the formation of large aggregates. Photometric performance issues such as these highlight the need for alternatives to traditional phosphors, and a suitable option seems to be the so-called luminophors.

In our work, we show that selected combinations of narrowemitter nanophosphors, made of colloidal quantum-dots, can be integrated into LEDs to achieve high-quality white light. In order to demonstrate the photometric efficiency of these devices, we used numerical simulations and experimental proofof-concept demonstrations. Our work suggests that high efficiencies can be achieved by using collective narrow emitters that allow for the tuning of the white light spectrum rather than continuous emitters.

The quality of white light can be evaluated using several photometric figures-of-merit. One of them is the color rendering index (CRI), which measures how efficiently a white light source reflects the real colors of an illuminated object. A good white light source possesses a CRI of 90 or above. An additional quality criterion is the luminous efficacy of optical radiation (LER), which represents the efficiency of the generated white spectrum in terms of the perception of the human eye. LER values rarely go above $3001 \mathrm{~m} / \mathrm{W}_{\text {opt }}$. The correlated color temperature (CCT) is another figure-of-merit used for quality evaluation. It indicates the temperature of a Planck black-body radiator whose perceived color most closely resembles that of the light-source.

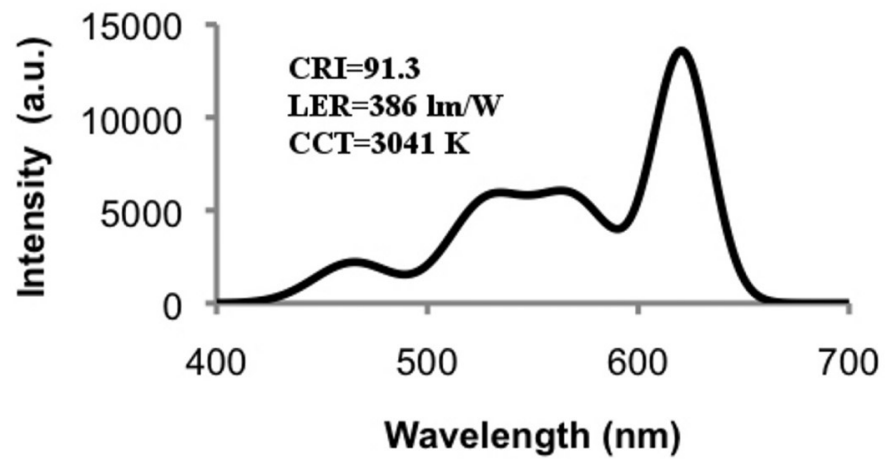

Figure 1. Spectrum generated using quantum-dot nanophosphors. The figures-of-merit CRI, LER, and CCT indicate the resulting photometric performance. $^{2}$

Warm white light has CCT values smaller that 3500K, which makes it highly desirable for indoor lighting applications. All of these performance criteria cannot be simultaneously satisfied using conventional broad-emitter phosphors. For instance, traditional phosphors can achieve high CRIs, but not in conjunction with high LERs.

To date, LER values of $2741 \mathrm{~m} / \mathrm{W}_{\text {opt }}$ are among the best reported performances of phosphor-based white LEDs. ${ }^{1}$ This is partially because phosphors do not have the ability to tune different shades of white while simultaneously achieving high photometric performances. White LEDs integrated with quantum-dot nanophosphors have the necessary spectral purity to enable their white-light spectrum to be tuned. However, the complex relationship between the photometric figures-of-merit makes it difficult to achieve large values of CRI and LER together with low values of CCT. This requires spectral design and the optimization of the nanophosphor quantum-dots.

Of all the colors forming white light, red is the most critical for determining photometric performance. ${ }^{2}$ Figure 1 shows a white-light spectra generated using a quantum-dot-based LED, with the resulting set of photometric figures-of-merit in the inset. Our results indicate that it is possible to obtain

Continued on next page 


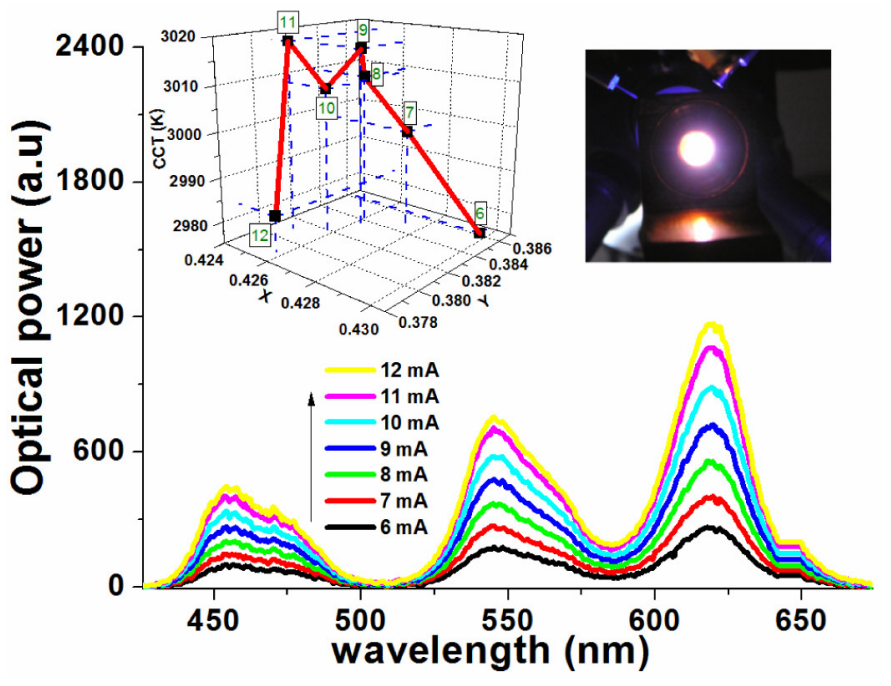

Figure 2. Electroluminescence spectra of the first quantum-dotbased white LED. This device integrates green, yellow, and orange-emitting quantum-dots-made of an inner core of cadmium selenide (CdSe) and an outer shell of zinc sulfide (ZnS)_into blue LEDs made of layers of indium gallium nitride ( $\mathrm{n} \mathrm{GaN}$ ) and gallium nitride (GaN). The plots show the collective emission and chromaticity properties for different current injection levels at room temperature. Left inset indicates different operating points in the chromaticity diagram, with the values inside the squares representing the current injection levels in $m A$. Each point has a CCT value, which is indicated in the z-axis coordinate. Right inset shows a quantum-dot-based LED generating white light. ${ }^{3}$

high-quality, spectrally efficient white light by using quantumdot nanophosphors. ${ }^{2}$

In previous works, we presented experimental demonstrations of spectrally efficient, high-quality white LEDs. ${ }^{3,4}$ For these proof-of-concept demonstrations, we integrated red and green quantum dots-made of an inner core of cadmium selenide (CdSe) and an outer shell of zinc sulfide ( $\mathrm{ZnS})$-into blue LEDs made of layers of indium gallium nitride (InGaN) and gallium nitride $(\mathrm{GaN})$. This combination gave us white LEDs with CRIs larger than 80, LERs larger than $3001 \mathrm{~m} / \mathrm{W}_{\text {opt }}$, and CCTs smaller than $3250 \mathrm{~K} .{ }^{4}$ By including an extra quantum-dot that emits in a different color, we achieved a four-color mixture that has a superior photometric performance (see Figure $2){ }^{3}$ In Figure 2, point 12 has coordinates $(x, y)=(0.425,0.378)$ in the chromaticity diagram, a CCT value of $2982 \mathrm{~K}$, a LER of $357 \mathrm{~lm} / \mathrm{W}_{\text {opt }}$, and a CRI equal to 89.2. To the best of our knowledge, this LED has the highest reported photometric performance, which demonstrates that narrow-emitter nanophosphors can exceed the photometric performance of the best reported phosphors.
We have shown that specifically designed quantum-dot nanophosphors can be integrated into LEDs. These devices produce high-quality white light whose photometric efficiency is verified using experimental proof-of-concept demonstrations. The high photometric capabilities, large quantum yields and well-established synthesis methodologies of these nanophosphors make them a suitable alternative to conventional phosphors. The challenge now is to make environmentally friendly (e.g. cadmium-free) quantum-dot nanophosphors with comparable quantum yields.

\section{Author Information}

\section{Hilmi Volkan Demir}

Departments of Electrical and Electronics Engineering and Physics

Institute of Materials Science and Nanotechnology (UNAM)

Bilkent University

Ankara, Turkey

and

Luminous! Center of Excellence for Semiconductor Lighting and Displays, Schools of Electrical and Electronic Engineering and Physical and Mathematical Sciences

Nanyang Technological University

Singapore

Hilmi Volkan Demir received his BSc from Bilkent University in 1998, and his PhD from Stanford University in 2004. He has contributed to the commercialization and licensing of several new technologies and startups in Turkey and France, which have led to more than 15 patent applications. He is the author of more than 70 publications in indexed peer-reviewed journals, and he has given more than 100 invited seminars. He is the recipient of national and international awards including the European Science Foundation European Young Investigator Award (ESFEURYI) and the Turkish Scientific and Technological Research Council Young Scientist Award (TUBITAK-TESVIK). He is a Fellow of Singapore's National Research Foundation. His research interests include high-quality white LEDs and innovative lightgeneration and harvesting.

\section{Talha Erdem and Sedat Nizamoglu}

Department of Electrical and Electronics Engineering Bilkent University

Ankara, Turkey 
Talha Erdem received his BSc in 2009 from Bilkent University, where he is currently studying for a MSc. His research is focused on the design and implementation of high-quality, quantumdot-integrated, white LEDs.

Sedat Nizamoglu is a PhD candidate in electrical and electronics engineering. He has published 29 papers in indexed journals and is a co-inventor in two pending patents. He has won several awards including the Student Innovation Award given by the European Technology Platform Photonics21, the Institute of Electrical and Electronics Engineers' Photonics Society Graduate Student Fellowship, and the SPIE Scholarship Award in Optical Science and Engineering.

\section{Xiao Wei Sun}

School of Electrical and Electronic Enineering.

Nanyang Technological University

Singapore, Singapore

From 1986 until 1994, Xiao Wei Sun studied for his BEng, MEng and $\mathrm{PhD}$ in photonics in Tianjin University, China. From 1994 to 1998, he pursued a second PhD in electrical engineering at the Hong Kong University of Science and Technology, Hong Kong. He is a SPIE and IoP Fellow and the recipient of the Nanyang Award for Research and Innovation 2009. He has co-authored over 250 publications in peer-reviewed journals.

\section{References}

1. M. R. Krames, O. B. Shchekin, R. Mueller-Mach, G. O. Mueller, G. Ling Zhou Harbers, and M. G. Craford, Status and future of high-power light-emitting diodes for solid-state lighting, J. Display Technol. 3 (2), pp. 160-175, 2007. doi:10.1109/JDT.2007.895339

2. T. Erdem, S. Nizamoglu, X. W. Sun, and H. V. Demir, A photometric investigation of ultra-efficient LEDs with high color rendering index and high luminous efficacy employing nanocrystal quantum dot luminophores, Opt. Express 18 (1), pp. 340-347, 2010. doi:10.1364/OE.18.000340

3. S. Nizamoglu, T. Erdem, X. W. Sun, and H. V. Demir, Warm-white light-emitting diodes integrated with colloidal quantum dots for high luminous efficacy and color rendering, Opt. Lett. 35 (20), pp. 3372-3374, 2010. doi:10.1364/OL.35.003372

4. S. Nizamoglu, G. Zengin, and H. V. Demir, Color-converting combinations of nanocrystal emitters for warm-white light generation with high color rendering index, Appl. Phys. Lett. 92 (3), pp. 031102-031104, 2008. doi:10.1063/1.2833693 